\title{
Investigations and system design for simultaneous energy and data transmission through inductively coupled resonances
}

\author{
C. Schmidt, E. Lloret Fuentes, and M. Buchholz \\ Research Group RI-ComET at the University of Applied Sciences Saarbrücken, Hochschul-Technologie-Zentrum, \\ Altenkesselerstr. 17/D2, 66115 Saarbrücken, Germany \\ Correspondence to: C. Schmidt (christian.schmidt@htwsaar.de)
}

Received: 12 December 2014 - Revised: 18 March 2015 - Accepted: 29 March 2015 - Published: 3 November 2015

\begin{abstract}
Wireless Power Transfer (WPT) with simultaneous data transmission through coupled magnetic resonators is investigated in this paper. The development of this system is dedicated to serve as a basis for applications in the field of Ambient Assisted Living (AAL), for example tracking vital parameters remotely, charge and control sensors and so on. Due to these different scenarios we consider, it is important to have a system which is reliable under the circumstance of changing positioning of the receiving device. State of the art radio systems would be able to handle this. Nevertheless, energy harvesting from far field sources is not sufficient to power the devices additionally on mid-range distances. For this reason, coupled magnetic resonant circuits are proposed as a promising alternative, although suffering from more complex positioning dependency.

Based on measurements on a simple prototype system, an equivalent circuit description is used to model the transmission system dependent on different transmission distances and impedance matching conditions. Additionally, the simulation model is used to extract system parameters such as coupling coefficients, coil resistance and self-capacitance, which cannot be calculated in a simple and reliable way.

Furthermore, a mathematical channel model based on the schematic model has been built in MATLAB $^{\odot}$. It is used to point out the problems occurring in a transmission system with variable transmission distance, especially the change of the passband's centre frequency and its bandwidth. Existing solutions dealing with this distance dependent behaviour, namely the change of the transmission frequency dependent on distance and the addition of losses to the resonators to increase the bandwidth, are considered as not inventive. First, changing the transmission frequency increases the complexity in the data transmission system and would use a dispro-
\end{abstract}

portional total bandwidth compared to the actually available bandwidth. Additionally, adding losses causes a decrease in the energy transmission efficiency.

Based on these facts, we consider a system that changes the channel itself by tuning the resonant coils in a way that the passband is always at a fixed frequency. This would overcome the previously described issues, and additionally could allow for the possibility to run several independent transmission systems in parallel without disturbing each other.

\section{Introduction}

The interest in WPT with coupled inductive resonators has grown in the last years, especially after the publications by Kurs et al. (2007) in Science. The nearfield coupling of resonating inductors promises the possibility to transfer an amount of energy over a mid-range distance quite efficiently compared to farfield approaches. Important aspects regarding the design of such a system have been in the focus of recent research, like coil design by Kim et al. (2012) and Lee et al. (2013), impedance match by Sample et al. (2010) and Cheon et al. (2011), coupled resonator's passband dependency on transfer distance by Sample et al. (2010) and Zhang et al. (2014) et cetera. So far, WPT systems have been described and investigated in depth, and solutions to overcome certain issues have been presented.

One of the most challenging properties is the transfer distance dependent passband characteristic of two or more coupled resonators. Different distances lead to changes in the coupling coefficient of the resonators, over-critical, critical and under-critical regions can be defined, similar to coupled, analog filters. The difficult thing here is that the transfer char- 
acteristic is not static. In principle, when thinking of a real application with freely movable wirelessly powered devices, it is a highly sophisticated task to predict the changes of the transfer function in a dynamic system.

This paper addresses the adaption of the system behaviour by changing the resonant coils parameters to keep the transfer maximum constant at one fixed frequency at first instance. Additionally, the data transmission as a new attribute, not considered in depth so far, adds some more restrictions to the system design, which are discussed based on the proposed channel stabilization method. Finally, the possibilities and challenges, especially concerning how to tune the coils, are highlighted. The study of the proposed system behaviour serves as a basis for further development regarding channel estimation and adaption. The proposed solution is adoptable for both, power and simultaneous data transfer. Other strategies like shifting the carrier frequency by Sample et al. (2010), which would complicate the data transmission, are considered as not useful in the target application. Also switching between a data and a power transfer mode as suggested by Kim et al. (2013) is assessed as a too complex solution in terms of synchronising both entities, especially in a very dynamic environment. Multi-band systems using two resonances like in Joanh et al. (2013) and Dionigi et al. (2012) are an interesting modification, using one frequency band for energy, another one for data transmission. The fact that resonators with two very well defined resonances must be used and, in our case, also controlled depending on the transfer distance, makes the implementation of a dynamically changeable system even more complex.

\section{Prototype measurements, parameter extraction and system modelling}

The prototype shown in Fig. 1 is used as a reference system in this paper. It is designed as a simple 4-coil-system similar to those described in Kurs et al. (2007); Kim et al. (2012, 2013); Cheon et al. (2011) and Awai et al. (2010). Additionally to the resonant coil, a single turn coupling coil is used on both source and load side to form an impedance matching network described in Sect. 2.2. This implementation of the impedance match is easy to build and to tune, and it has been shown to be a good compromise between transfer distance and efficiency (Hui et al., 2013).

Mechanically, each resonant coil has a coupling coil attached to it. The distance between both can be varied to change the coupling factor and, due to this, the impedance match. Both resonant coils with their coupling coil can be moved on a fixed rail, making it possible to change the transfer distance while keeping the impedance match untouched. All coils are arranged concentrically.

The resonant coils are each wound on a piece of polypropylene tube with a radius of $55 \mathrm{~mm}$, in which a notch of $0.5 \mathrm{~mm}$ was milled to keep the windings in a constant in-

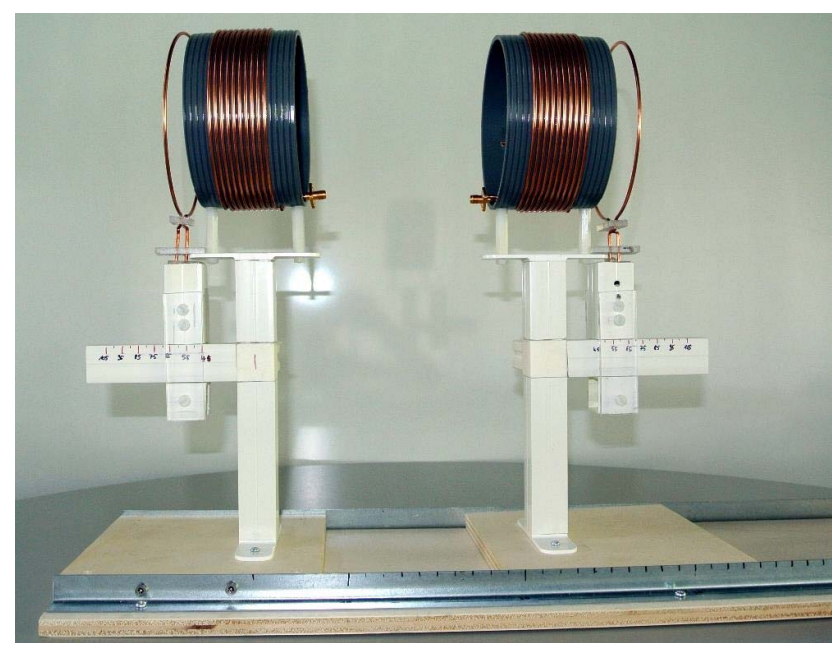

Figure 1. Wireless Power Transfer prototype used in this work.

clination of $3.5 \mathrm{~mm}$ per turn. The copper wire diameter is of $1.12 \mathrm{~mm}$, resulting in a total coil radius of $55.63 \mathrm{~mm}$.

Similar to the system presented in Kurs et al. (2007), the resonant coils are initially used in their self-resonance. The design goal was to achieve a self-resonant frequency of approximately $20 \mathrm{MHz}$. To ensure this, each coil consists of ten windings, promising enough self-capacitance for a low resonant frequency. CST Microwave Studio $^{\circledR}$ was used to validate this choice. The simulation shows a resonance at $21.78 \mathrm{MHz}$ and an inductance of $14.775 \mu \mathrm{H}$ at $10 \mathrm{MHz}$. At frequencies closer to the resonance, the inductance values extracted from the simulated $Z$ parameters are not meaningful because the coil's behaviour near its resonance frequency can no longer be considered as inductive. The inductance value was validated using Rayleigh and Niven's formula, Eq. (1), for coils short compared to their diameter; afterwards, Rosa's current sheet correction, $\Delta L$, is applied (Rosa et al., 1916):

$$
\begin{aligned}
L_{\mathrm{S}} & =4 \pi a n^{2}\left[\log \frac{8 a}{b}-\frac{1}{2}+\frac{b^{2}}{32 a^{2}}\left(\log \frac{8 a}{b}+\frac{1}{4}\right)\right] \\
\Delta L & =4 \pi a n(A+B) .
\end{aligned}
$$

Finally, this results in the corrected inductance value $L$ :

$L=L_{s}+\Delta L$

In these formulas, $a$ is the winding diameter, $b$ is the total height of the coil, $n$ is the number of turns, $A$ and $B$ are correction values tabulated by Rosa et al. (1916). For our resonant coils, this formula gives a corrected inductance value of $14.7 \mu \mathrm{H}$, which is in absolute agreement with the simulated value. Using the well-known Thomson formula, we calculate the coil's self capacitance by setting $f=21.7 \mathrm{MHz}$, neglecting any loss, and $L=14.7 \mu \mathrm{H}$ resulting in a capacitance of 


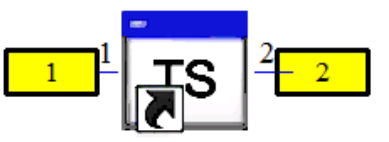

C60_R10.s2p

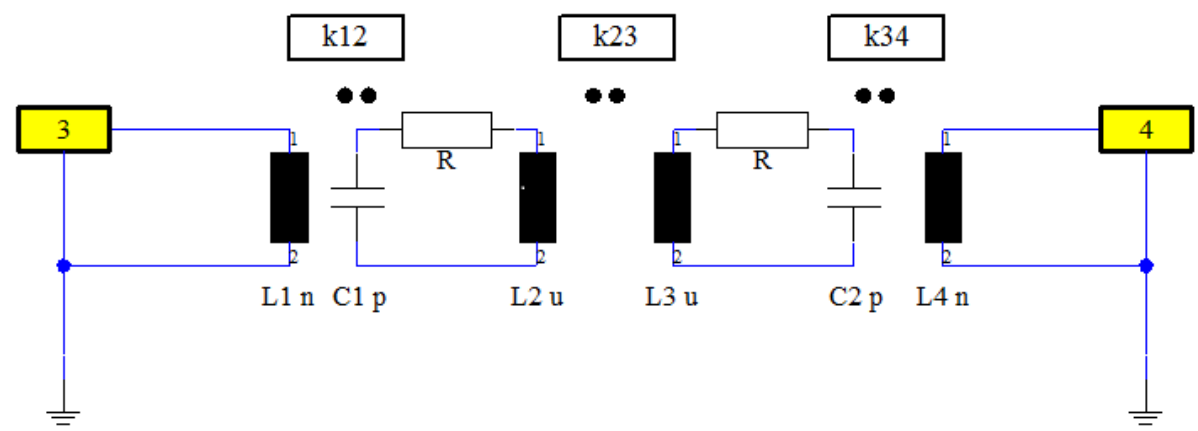

Figure 2. CST Design Studio model used for parameter extraction.
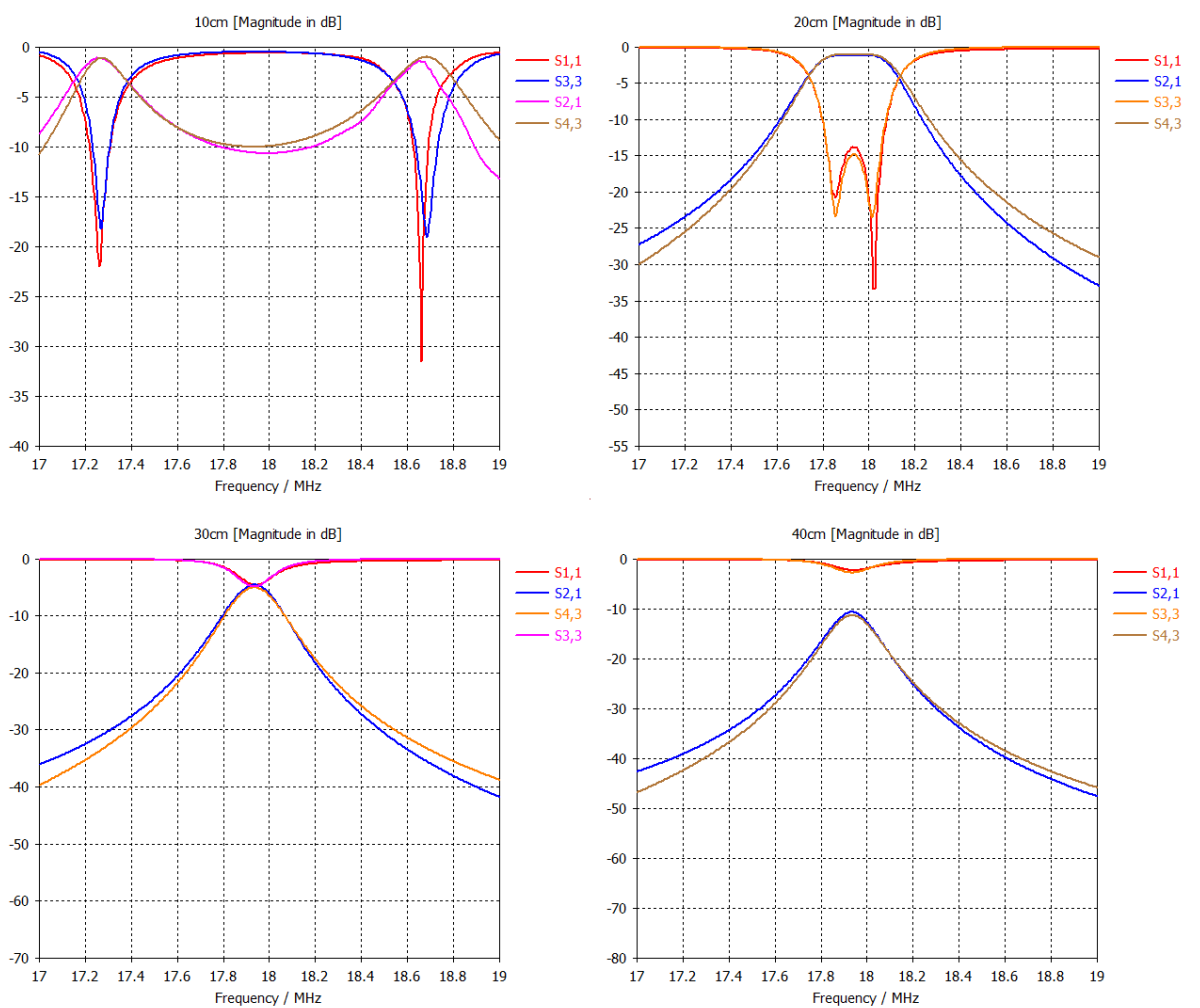

Figure 3. Measured $\left(S_{1,1}, S_{2,1}\right)$ and simulated $\left(S_{3,3}, S_{4,3}\right)$ transmission and reflection amplitudes.

$3.63 \mathrm{pF}$.

$f=\frac{1}{2 \pi \sqrt{\mathrm{LC}}}$

In order to be able to access the resonant coils directly, SMA connectors are attached to each of them. Thus, the coils can directly be measured and additional variable capacitors can be attached to tune them.

\subsection{Measurements and parameter extraction}

First measurements on the prototype showed that the expected resonant frequency of $21.78 \mathrm{MHz}$ was shifted to $17.92 \mathrm{MHz}$ for both coils. The measured inductance of the resonant coils is $14.56 \mu \mathrm{H}$ at $1 \mathrm{MHz}$, which is a tolerable result compared to the calculated and simulated value of 14.7 $\mu \mathrm{H}$. As a consequence, the shifted resonant frequency 
Table 1. Lumped elements values.

\begin{tabular}{ccccc}
\hline$L_{2}, L_{3}$ & $C_{1}, C_{2}$ & $R$ & $k_{1,2}, k_{3,4}$ & $L_{1}, L_{4}$ \\
\hline $14.56 \mu \mathrm{H}$ & $5.458 \mathrm{pF}$ & $2.8 \Omega$ & 0.151 & $312 \mathrm{nH}$ \\
\hline
\end{tabular}

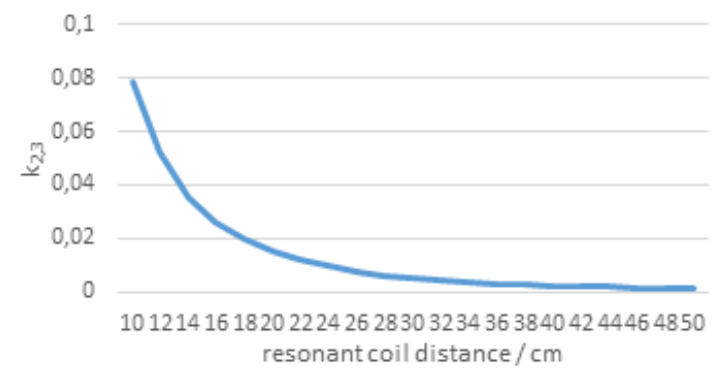

Figure 4. Coupling factor $k_{2,3}$ over transfer distance in $\mathrm{cm}$.

must be the result of an increased capacitance value of approximately $5.4 \mathrm{pF}$ and/or additional losses compared to the simulation. The material model for polypropylene and the mechanical tolerances of the wires to the connectors are the most likely candidates for an uncertainty like this.

Due to a direct measurement of the coil's inductance, capacitance and loss resistance at the resonant frequency is not feasible, a parameter extraction based on measured data as Touchstone $^{\circledR}$ files and a schematic model was performed.

As a first step, measurements were conducted on the full 4-coil-system with constant matching condition (position of the coupling coils on both sides at $6 \mathrm{~cm}$ apart from the resonant coils) and transfer distances from 10 to $50 \mathrm{~cm}$ in steps of $2 \mathrm{~cm}$.

The equivalent circuit shown in Fig. 2 has been widely used to characterize WPT systems. Although cross and capacitive couplings between the coils as well as capacitance respectively loss resistance of the coupling coils are neglected, good agreement between measured and simulated responses have been reported in Sample et al. (2010) and Dionigi et al. (2011). Due to this, the model is used to extract the circuit parameters by means of an optimizer run for every transfer distance we measured. Figure 3 shows the measured response and the optimized schematic simulation for four different transfer distances. For the first run $(10 \mathrm{~cm}$ resonant coil distance, $6 \mathrm{~cm}$ coupling coil distance), the schematic values were initially set. The inductance value was taken from the measurements, the capacitance from the simplified calculation using Thomson's formula and the measured resonance frequency. Coupling factors and resistive losses were adapted until a certain fitting was achieved. Then, an optimizer was set up changing capacitance, resistance and coupling factors until the difference between the $S 21$-parameter of measured data and schematic simulation was below a threshold of 0.02 (linear scaling). For the following runs, the measured data file was updated to the next higher transfer distance and the same

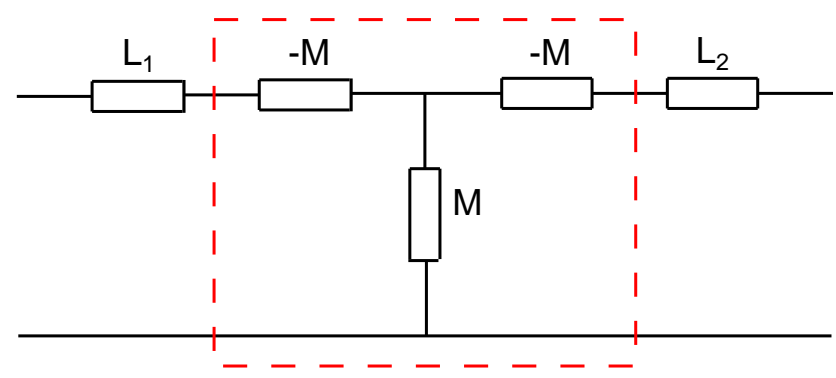

Figure 5. Impedance inverter.

optimizer was used with the same goal; however, just the coupling factor between the resonant coils was changed (results shown in Fig. 3). The fact that a fit between both curves has been realized shows that the schematic model works fine for describing the system.

Additionally, the extracted parameters tabulated below have proven to be constantly a good approximation of the real physical model. In Table $1, L_{2}$ and $L_{3}$ stand for resonant coil inductance, $C_{1}$ and $C_{2}$ for self-capacitance of the resonant coils, $R$ for loss resistance of the resonant coils, $k_{1,2}$ and $k_{3,4}$ the coupling factor between coupling and resonant coils and $L_{1}$ and $L_{4}$ for coupling coil inductances.

The extracted coupling factor between the resonant coils, $k_{2,3}$, is shown in Fig. 4, dependent on the transfer distance. Based on the full set of schematic component values, the following section describes a mathematical model used to describe the transfer channel.

\subsection{System modelling}

Looking again at Fig. 2, which we used to extract the coil's parameters, we can see that there are three pairs of coupled inductors. A pair of coupled inductors, like the ones shown in Fig. 2, can also be represented by means of a T-equivalent circuit. This T-equivalent circuit behaves as an impedance inverter, formed by the three mutual inductances $M$ shown in Fig. 5 (Tosic et al., 2006). An impedance inverter as described below has the following ABCD matrix:

$\mathbf{A B C D}=\left[\begin{array}{cc}0 & -j K_{1,2} \\ \frac{-j}{K_{1,2}} & 0\end{array}\right]$.

In the above matrix, the inverter constant $K$ is equal to $K_{1,2}=\omega M=\omega k_{1,2} \sqrt{L_{1} L_{2}}$. In this equation, $k_{1,2}$ is the coupling factor between the coils $L_{1}$ and $L_{2}, M$ is the mutual inductance. Replacing the three pairs of coupled inductors by their corresponding impedance inverters and considering the internal resistance and capacitance of every inductor, we end up in a circuit like the one presented in Fig. 6. 


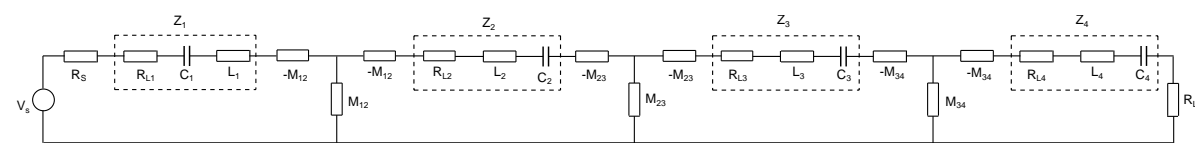

Figure 6. Four coil equivalent circuit.

We can analyse the transfer function of such a circuit by using a cascade of ABCD matrices. Thus, we obtain:

$$
\begin{aligned}
\mathbf{A B C D}=\left[\begin{array}{cc}
1 & Z_{1} \\
0 & 1
\end{array}\right] & \times\left[\begin{array}{cc}
0 & -j K_{1,2} \\
\frac{-j}{K_{1,2}} & 0
\end{array}\right] \times\left[\begin{array}{cc}
1 & Z_{2} \\
0 & 1
\end{array}\right] \\
& \times\left[\begin{array}{cc}
0 & -j K_{2,3} \\
\frac{-j}{K_{2,3}} & 0
\end{array}\right] \times\left[\begin{array}{cc}
1 & Z_{3} \\
0 & 1
\end{array}\right] \\
& \times\left[\begin{array}{cc}
0 & -j K_{3,4} \\
\frac{-j}{K_{3,4}} & 0
\end{array}\right] \times\left[\begin{array}{cc}
1 & Z_{4} \\
0 & 1
\end{array}\right]
\end{aligned}
$$

A similar analysis has been performed in Dionigi et al. (2011) with equivalent results. If we now consider that the system is symmetric:

$$
\begin{aligned}
Z_{1} & =Z_{4} \\
Z_{2} & =Z_{3} \\
K_{1,2} & =K_{3,4}
\end{aligned}
$$

Then, the ABCD matrix gets simplified:

$$
\begin{aligned}
& \mathbf{A B C D}= \\
& {\left[\begin{array}{cc}
j \frac{Z_{1} Z_{2}^{2}+K_{1,2}^{2} Z_{2}+Z_{1} K_{2,3}^{2}}{K_{1,2}^{2} K_{2,3}} & \frac{Z_{1}^{2} Z_{2}^{2}+K_{2,3}^{2} Z_{1}^{2}+2 K_{1,2}^{2} Z_{1} Z_{2}+K_{1,2}^{4}}{K_{1,2}^{2} K_{2,3}} \\
j \frac{Z_{2}^{2}+K_{2,3}^{2}}{K_{1,2}^{2} K_{2,3}} & j \frac{Z_{1} Z_{2}^{2}+K_{1,2}^{2} Z_{2}+Z_{1} K_{2,3}^{2}}{K_{1,2}^{2} K_{2,3}}
\end{array}\right]}
\end{aligned}
$$

In order to calculate the scattering matrix parameter $S_{2,1}$, we can apply the well-known transformation in Eq. (8) from Hui et al. (2013), where $Z_{0}=50 \Omega$

$S_{2,1}=\frac{2(A D-B C)}{A+\frac{B}{Z_{0}}+C Z_{0}+D}$

For the sake of verification, we compare the behaviour of the above formula with the schematic simulations. In Fig. 7, we can see the frequency response of a four coils configuration where the resonant coils are $20 \mathrm{~cm}$ apart from each other, the coupling coils and the resonant coils are separated $6 \mathrm{~cm}$ from each other. For these particular distances, the coupling factors are $k_{1,2}=k_{3,4}=0.151$ and $k_{2,3}=0.0156073$.

\section{Channel modelling}

In the last two sections, we first extracted all parameters for a simplified schematic model describing our prototype system, based on measurements at discrete distances. Afterwards, we
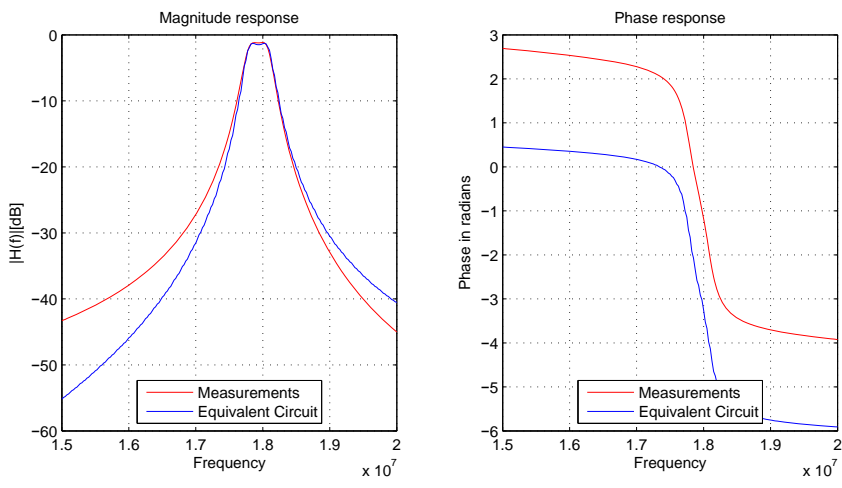

Figure 7. Measurement results vs. equivalent circuit calculation.

found the transfer function shown in Eq. (8) which describes the system behaviour as a simple equation. As this description has proven to be accurate, we are also able to model and predict transfer distances not considered in our measurements.

This development allows us to switch from a physical description in terms of lumped element values to a mathematical model. We use a Finite Impulse Response (FIR) filter to model the transfer in MATLAB ${ }^{\odot}$ to be able to analyse arbitrary data transfer through the channel.

Since we have already an expression for the $S_{2,1}$ parameter, we can easily obtain the frequency response of such a hypothetical filter by simple substitution of the lumped element values in Eq. (8) by those extracted from the measurements. Furthermore, it is known that the frequency response of a filter is just the Fourier Transform of its impulse response $h[n]$ (Oppenheim et al., 1989). Therefore, using the Inverse Discrete Fourier Transform (IDFT, Eq. 9), we can obtain the impulse response of a channel described by Eq. (7).

$h[n]=\frac{1}{2 \pi} \int_{-\pi}^{\pi} H\left(e^{j \omega}\right) e^{j \omega n} d \omega$

In case of a FIR filter, the impulse response values are simply the filter's coefficients, as shown in Eq. (10). The IDFT of Eq. (7) values can therefore be directly used as filter coefficients.

$y[n]=\sum_{k=0}^{N-1} b_{k} x[n-k] \quad h[n]=\sum_{k=0}^{N-1} b_{k} \delta[n-k]$

Using Eq. (7) and the IDFT, we can now calculate the filter coefficients and compare the transfer functions in Fig. 8. 

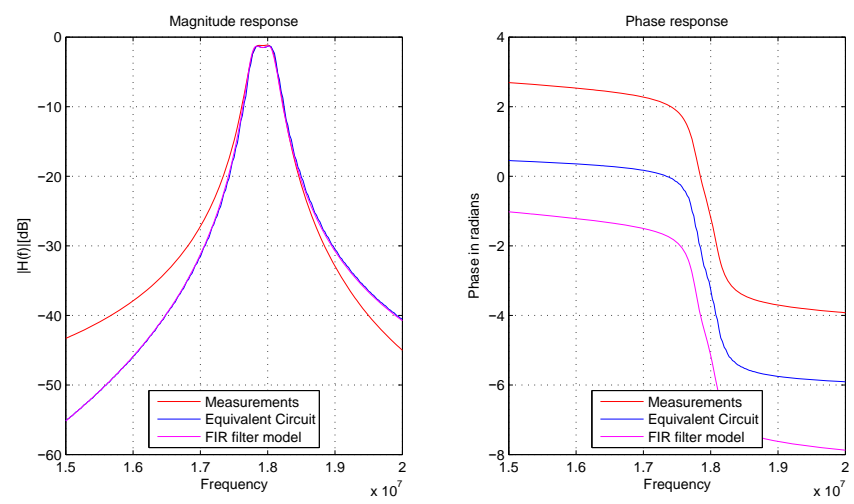

Figure 8. Measurement results vs. equivalent circuit and FIR filter model analysis.

Apart from a constant phase deviation, the FIR filter modelling provides a good approximation for the four coils' frequency response. Having obtained the filter's coefficients will allow us to perform deeper analysis of this kind of communication channels, as well as the evaluation of possible solutions for the challenges it may present. In order to give an idea of what these simulations might look like, we study the following example. We generate a spectrum-shaped pseudo random baseband signal which modulates a $17.935 \mathrm{MHz}$ carrier as Quadrature Phase-Shift Keying (QPSK), as this is the resonant frequency of each single resonator. This results in a $125 \mathrm{KHz}$ RF bandwidth. Then, we send it through a FIR filter with the coefficients we just extracted, and finally we mix down the signal to the baseband and demodulate it. As expected, varying the distance between the coils produces various effects, since they represent different channels.

Assuming that the coupling coils remain in a fixed position with respect to the resonant coils, the first and most obvious effect is resonant frequency splitting, occurring when the resonant coils are over-critically coupled. In this region, the closer the two resonant coils are, the more deviation from the centre appears between the two new resonant frequencies. Moving away the two resonant coils, until a particular distance is reached, causes the two resonant frequencies to merge in a single one (critical coupling). At this point, we obtain the minimum attenuation at the resonant frequency as well as the widest $3 \mathrm{~dB}$ bandwidth. Beyond this distance, the attenuation increases while the $3 \mathrm{~dB}$ bandwidth may decrease, mainly due to impedance mismatch. Constant adaption of the impedance match can be a first attempt to improve the transmission as highlighted in Sample et al. (2010), and should be applied in addition to the proposal presented in the next section.

In the following figures, a constellation diagram is shown next to the transfer function. It serves as a qualitative measure of the data transmission performance. A totally undistorted, ideal QPSK signal would produce a diagram with one discrete point in every quadrant (absolute value $|I(t)|=$ $|Q(t)|=1$ ), representing the four signal states.

Figure 9 shows as an example the transfer function for a distance of $10 \mathrm{~cm}$ and the corresponding constellation diagram for the data signal described above. As we can see, mainly the signal's amplitude decreases to a normalised value of around 0.2 , corresponding to the attenuation of $11.5 \mathrm{~dB}$. The signal phase is slightly rotated, but the constellation points form quite dense spots.

At an increased distance of $20 \mathrm{~cm}$ (Fig. 10), the amplitude is higher than before. This is due to the fact that our signal transmission frequency is now right in the middle of the channel's maximum. The two resonators are no more overcritically coupled but nearly critical and depict an attenuation of $1.5 \mathrm{~dB}$. On the other hand, the constellation points form a less dense cloud, mainly caused by linear phase distortion or in other words a non-constant group delay.

Finally, at $30 \mathrm{~cm}$ transfer distance (Fig. 11), both effects described above occur. The constellation diagram shows even wider spread points with a small corresponding amplitude caused by the amplitude and phase distortion induced by the channel.

As we have seen, for data transmission, an untuned system of coupled resonators forms a very dynamic, distance dependent channel. All of the individual data signals described by the constellation plots above can possibly be demodulated by a digital signal processing system. But for a dynamic system, the range of different channels would be too high. In this particular channel, we have the ability to tune the channel for every operating condition. Like this, we can optimize not only the data channel, but also maintain a constant maximum power transfer.

\section{Channel adaption}

Summing up the last section, by varying the transfer distance, the channel characteristic of the coupled resonators changes considerably in amplitude and phase. To handle this behaviour, we suggest the implementation of an automated, adaptive channel tuning dependent on the actual distance.

Recent research has been carried out by different groups to address the problem of channel dependency on the transfer distance in pure energy transmission systems. Typical experimental implementations to decrease the channel variation make use of additional impedance matching networks attached to the transfer system (Beh et al., 2013; Waters et al., 2012; Sample et al., 2013) or several switchable coupling coils (Kim et al., 2012). Especially (Sample et al., 2013) shows, that this impedance matching network adds additional resonant peaks in between the resonators main peaks, thus providing the possibility to transfer energy at a fixed frequency. Due to the fact that the matching networks consist of switchable elements, it is obvious that the system performance is optimized only for discrete distances dependent 


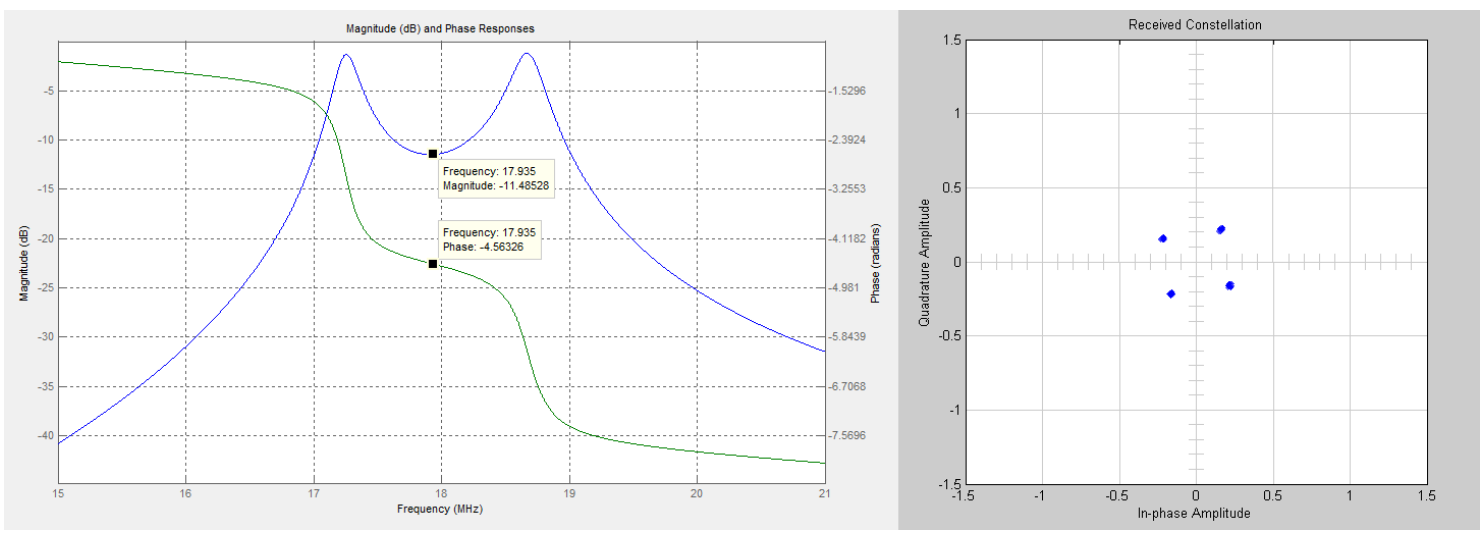

Figure 9. FIR-filter magnitude/phase and constellation plot for $10 \mathrm{~cm}$ distance.

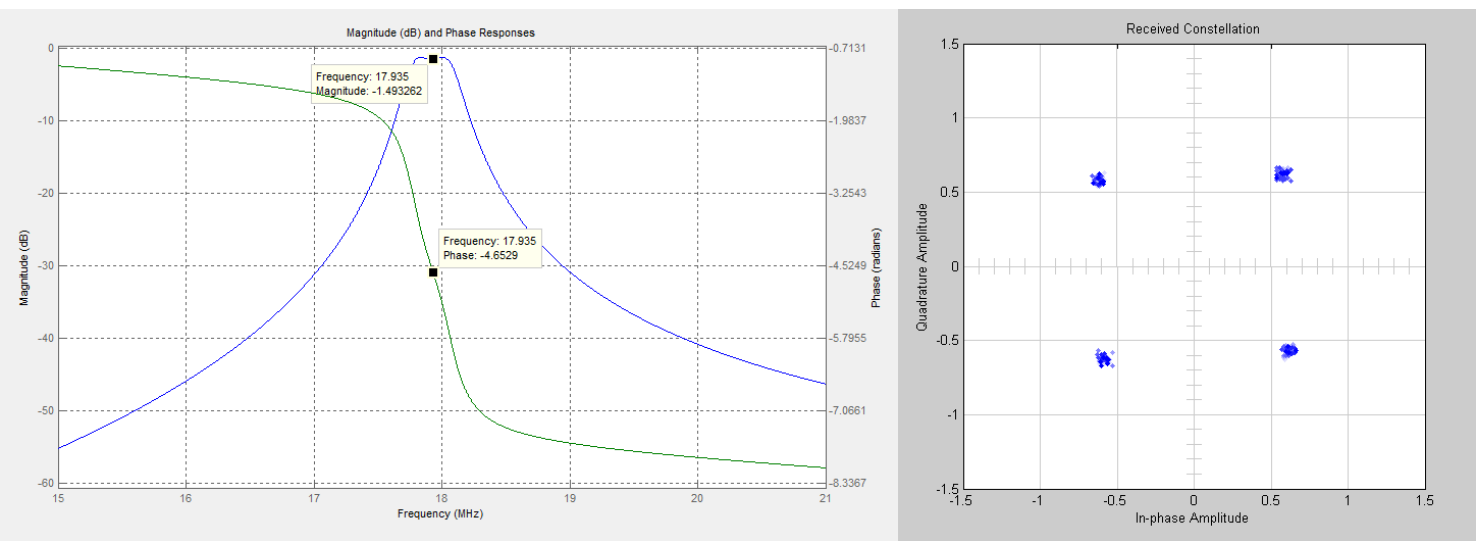

Figure 10. FIR-filter magnitude/phase and constellation plot for $20 \mathrm{~cm}$ distance.

on the number of possible switch combinations. Continuous matching networks are presented in (Beh et al., 2010) and (Jang et al., 2012), in which the first system uses motor controlled variable air capacitors, which is certainly limited to large-scale applications like electric vehicles. The second paper reports an impedance match implementation based on varactors. These devices would be also very convenient as tuning elements, unfortunately, their RF voltage handling capability is very limited. Using varactors as tuning elements for the resonators is not useful because of the quite high voltages (tens to hundreds of volts) at the coil's terminals, even for small or mid-range power levels.

Regarding data transmission, the impedance matching network approach introduces again a channel variation due to the different matching states dependant on transfer distance. Additionally, the performance of matching network based implementations degrades compared to a system which shifts the transfer frequency according to the channel response (Sample et al., 2013). Although we stated in the introduction, that frequency shifting is not an option for a data transmission system, it can be used as a reference for other implemen- tations because it involves the least elements and therefore promises maximum performance.

Based on this fact, tuning the channel response dependent on transfer distance can be considered as counterpart to frequency shifting. A first implementation of such a system has recently been presented in Ricketts et al. (2013). A bank of switchable capacitors is used to shift the resonant frequencies of the coils in a way that the resulting channel shows a transfer maximum always at a fixed frequency. Like this, the channel's amplitude and phase behaviour is a lot less dependent on transfer distance and more stable compared to the impedance matching approach.

To validate the benefits of this approach, we modified our simulation model by increasing the resonators capacitance to locate the lower peak of the transfer function always at $17 \mathrm{MHz}$ which is just below the lower resonant mode for $10 \mathrm{~cm}$ distance (see Fig. 9). Now, by adding a capacitance of $0.165 \mathrm{pF}$, we are able to tune the channel's lower passband to exactly our centre frequency. We did the same for larger distances with appropriately adapted capacitance values. As shown in Fig. 12, again for the case of $10 \mathrm{~cm}$ transfer distance, the attenuation has been drastically reduced, while 


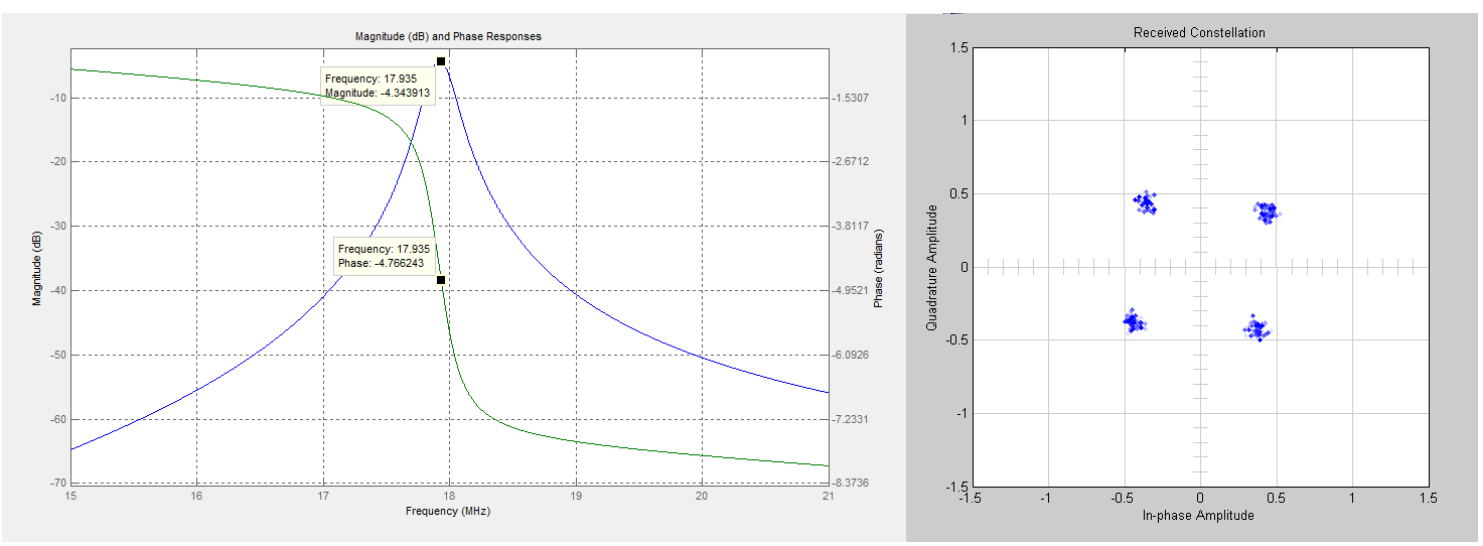

Figure 11. FIR-filter magnitude/phase and constellation plot for $30 \mathrm{~cm}$ distance.
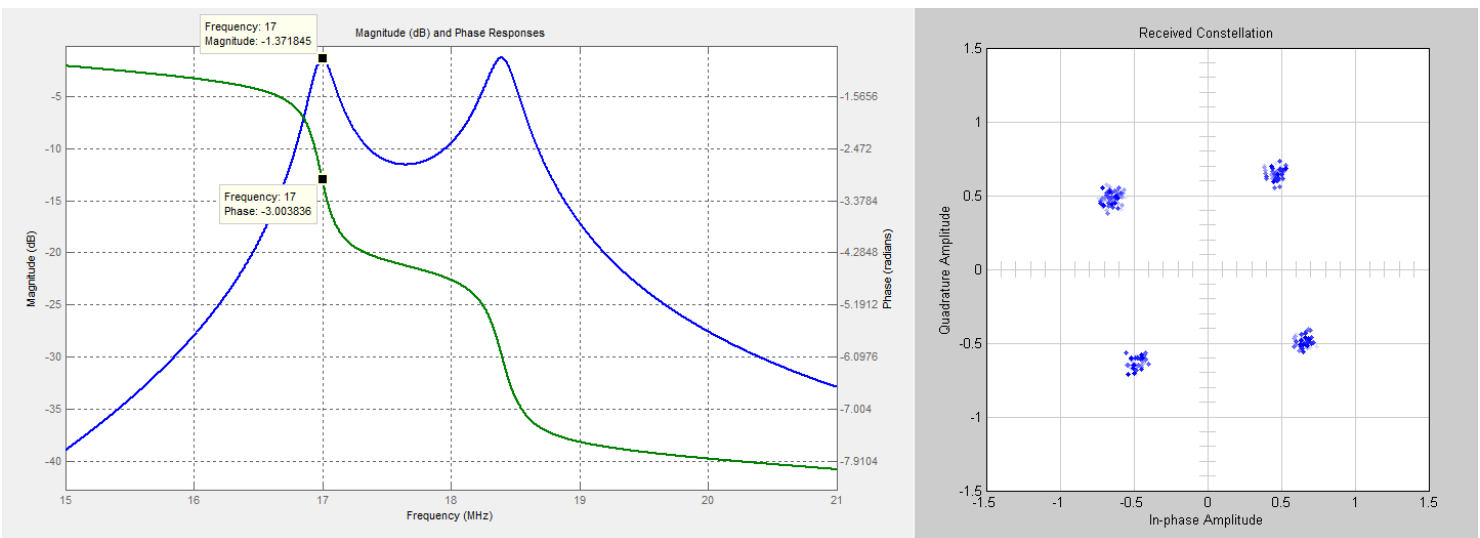

Figure 12. Tuned FIR-filter magnitude/phase response and constellation plot for $10 \mathrm{~cm}$ distance.

the linear distortion introduced due to the sharp frequency response around $17 \mathrm{MHz}$ has increased. Anyway, a proper demodulation is still possible.

If this channel adaption scheme is used for all resonant coil distances, the variation of the channel behaviour is minimized. Table 2 shows simulation results, where the error vector magnitude (EVM) has been calculated for different resonant coil separations in case of untuned and capacitively tuned resonant coils. The EVM is defined in different standards as the percentage ratio of the RMS error to the square root of average constellation power.

As can be seen from these results, the channel tuning results in a stable performance with a maximum of $20 \%$ EVM and just a small variation over distance. For distances higher than approximately $20 \mathrm{~cm}$, the system is no longer in the over-critically coupled region (see also Sect. 3). Subsequently, the capacitance value to tune the passband to $17 \mathrm{MHz}$ stays constant. Anyway, the EVM increases because of higher transfer losses and reduced bandwidth in the undercritically coupled regime.

As a result, a couple of tuned coils greatly simplifies the digital signal processing required to recover the transmitted
Table 2. EVM calculation after simulation results.

\begin{tabular}{lccc}
\hline $\begin{array}{l}\text { Resonant } \\
\text { coil distance }\end{array}$ & $\begin{array}{c}\text { Untuned } \\
\text { coils }\end{array}$ & $\begin{array}{c}\text { Tuned } \\
\text { coils }\end{array}$ & $\begin{array}{c}\text { Added } \\
\text { capacitance }\end{array}$ \\
\hline $10 \mathrm{~cm}$ & $73.33 \%$ & $20.18 \%$ & $0.165 \mathrm{pF}$ \\
$12 \mathrm{~cm}$ & $60.86 \%$ & $20.12 \%$ & $0.319 \mathrm{pF}$ \\
$14 \mathrm{~cm}$ & $45.82 \%$ & $19.90 \%$ & $0.420 \mathrm{pF}$ \\
$16 \mathrm{~cm}$ & $32.65 \%$ & $18.90 \%$ & $0.472 \mathrm{pF}$ \\
$18 \mathrm{~cm}$ & $22.04 \%$ & $18.15 \%$ & $0.524 \mathrm{pF}$ \\
$20 \mathrm{~cm}$ & $15.72 \%$ & $16.70 \%$ & $0.605 \mathrm{pF}$ \\
$30 \mathrm{~cm}$ & $43.21 \%$ & $43.15 \%$ & $0.605 \mathrm{pF}$ \\
\hline
\end{tabular}

data. In addition, simultaneous efficient energy transfer is possible, since the transmission is now always at the lower resonant mode's frequency, resulting in increased efficiency. In Fig. 12, $S_{21}$ peak magnitude at $17 \mathrm{MHz}$ is just $-1.37 \mathrm{~dB}$ in contrast to $-11.49 \mathrm{~dB}$ in Fig. 9.

To achieve this goal, basic system aspects still have to be examined. First, a method has to be found to perform a fast and reliable channel estimation to gather the information of the current channel status. Second, based on this, the res- 
onators on source and load side have to be tuned. To change the passband's centre to $17 \mathrm{MHz}$ in Fig. 12, a parallel capacitance of $0.165 \mathrm{pF}$ had to be added in the simulation. In a real system, the absolute value of the tuning capacitor can be higher, dependant on the resonator design and the desired transfer frequency. On the other hand, the capacitance values shown in Table 2 highlight that a tuning range of about $500 \mathrm{fF}$ has to be realized with a precision in the range of $10 \mathrm{fF}$ for our prototype system. For comparable system, at least the precision will be in the same range. Such a tuning could for example involve capacitors inductively coupled to the resonators as described in Ricketts et al. (2014). However, further research is required to find a reliable and precise tuning technique.

\section{Conclusions}

In this paper, we present a system concept for simultaneous energy and data transfer analysis. Based on a prototype system, measurements and parameter extraction using simulations are carried out. An equivalent circuit is validated and embedded in a channel modelling in MATLAB ${ }^{\odot}$. The impact of a varying channel on the data and energy transmission is analysed. Regarding a real-life application, a method to tune the channel behaviour is proposed, which promises an optimal, distance-independent transfer in a wide range. Further research has to be done on certain aspects regarding a useful implementation.

\section{The Supplement related to this article is available online at doi:10.5194/ars-13-217-2015-supplement.}

Acknowledgements. This paper presents results from the first phase of the research project "ComPad - Smart Modem zur kontaktlosen Sensordaten- und Bildübertragung bei gleichzeitiger Energieaufladung für eine alterssensible Anpassung der hauslichen Infrastruktur". The project is funded by the German Federal Ministry of Education and Research (BMBF), through the "IngenieurNachwuchs" funding stream (young engineers).

Edited by: M. Chandra

Reviewed by: two anonymous referees

\section{References}

Awai, I. and Ishida, T.: Design of Resonator Coupled Wireless Power Transfer System by Use of BPF Theory, J. Korean. Inst. Electrom. Eng. Sci., 10, 237-243, 2010.

Beh, T. C., Kato, M., Imura, T., and Hori, Y.: Wireless Power Transfer System via Magnetic Resonant Coupling at Fixed Resonance Frequency-Power Transfer System Based on Impedance Matching, World Electr. Vehicle J., 4, 744-753, 2010.
Beh, T. C., Kato, M., Imura, T., Oh, S., and Hori, Y.: Automated Impedance Matching System for Robust Wireless Power Transfer via Magnetic Resonance Coupling, IEEE T. Ind. Electron., 60, 3689-3698, 2013.

Cheon, S., Kim, Y, Kang, S., Lee, M. L., Lee, J., and Zyung, T.: Circuit-Model-Based Analysis of a Wireless Energy-Transfer System via Coupled Magnetic Resonances, IEEE T. Ind. Electron., 58, 2906-2914, 2011.

Dionigi, M. and Mongiardo, M.: CAD of Efficient Wireless Power Transmission Systems, 2011 IEEE MTT-S, 2011 IEEE MTTS International Microwave Symposium, Baltimore Convention Center, USA, 5-10 June 2011, 1-4, 2011.

Dionigi, M. and Mongiardo, M.: A novel resonator for simultaneous Wireless Power Transfer and Near Field Magnetic Communications, 2012 IEEE MTT-S, 17-22 June 2012, 1-3, 2012.

Hui, S. Y. R, Zhong, W., and Lee, C. K.: A Critical Review of Recent Progress in Mid-Range Wireless Power Transfer, IEEE T. Power Electr., 29, 4500-4511, 2013.

Jang, B.-J., Lee, S., and Yoon, H.: HF-Band Wireless Power Transfer System: Concept, Issues, and Design, Prog. Electromagn. Res., 124, 211-231, 2012.

Jonah, O., Georgakopoulos, S. V., and Yao, S.: Strongly Coupled Resonance Magnetic for RFID Applications, IEEE Apsursi., 1110-1111, 2013.

Kim, J., Son, H., Kim, D., and Park, Y.: Optimal Design of a Wireless Power Transfer System with Multiple Self-Resonators for an LED TV, IEEE T. Consum. Electr., 58, 775-780, 2012.

Kim, J., Choi, W.-S., and Jeong, J.: Loop Switching Technique for Wireless Power Transfer using Magnetic Resonance Coupling, Prog. Electromagn. Res., 138, 197-209, 2013.

Kurs, A., Karalis, A., Moffatt, R., Joannopoulos, J. D., Fisher, P., and Soljačić, M.: Wireless Power Transfer via Strongly Coupled Magnetic Resonances, Science, 317, 83-86, 2007.

Lee, G., Waters, B. H., Shi, C., Park, W. S., and Smith, J. R.: Design considerations for asymmetric magnetically coupled resonators used in wireless power transfer applications, 2013 IEEE Radio and Wireless Symposium (RWS), 2013 IEEE Radio \& Wireless Week, Renaissance Austin, USA, 20-23 January 2013, 328-330, 2013.

Oppenheim, A. V. and Schafer, R. W.: Discrete-Time Signal Processing, International Edition, Prentice Hall, USA, 1989.

Ricketts, David S., Chabalko, Matthew J., and Hillenius, Andrew: Optimization of Wireless Power Transfer for Mobile Receivers Using Automatic Digital Capacitance Tuning, Proceedings of the 43rd European Microwave Conference, 515-518, 2013.

Ricketts, D. S., Chabalko, M. J., and Hillenius, A.: Tri-Loop Impedance and Frequency Matching With High-Q Resonators in Wireless Power Transfer, IEEE Antenn. Wirel. Pr., 13, 341-344, 2014.

Rosa, E. B. and Grover, F. W.: Formulas and Tables for the Calculation of Mutual and Self-Inductance [Revised], Sci. P. Bur. Stand., 169, 116-122, 1916.

Sample, A. P., Meyer, D. A., and Smith, J. R.: Analysis, Experimental Results, and Range Adaptation of Magnetically Coupled Resonators for Wireless Power Transfer, IEEE T Ind. Electron., 58, 544-554, 2010.

Sample, A. P., Waters, B. H., Wisdom, S. T., and Smith, J. R.: Enabling Seamless Wireless Power Delivery in Dynamic Environments, Proceedings of the IEEE, 101, 1343-1358, 2013. 
Tosic, D. V. and Potrebic, M.: Symbolic analysis of immittance inverters, 14th Telecommunication Forum, Belgrade (Serbia), 2123 November 2006, 584-587, 2006.

Waters, B. H., Sample, A. P., and Smith, J. R.: Adaptive Impedance Matching for Magnetically Coupled Resonators, Pr. Electromagn. Res. S., 694-701, 2012
Zhang, Y. and Zhao, Z: Frequency splitting Analysis of Two-Coil Resonant Wireless Power Transfer, IEEE Antenn. Wirel. Pr., 13, 400-402, 2014. 